\title{
Active and passive opening of the cardia and its relation to the pathogenesis of hiatus hernia
}

\author{
H. DAINTREE JOHNSON \\ From the Postgraduate Medical School of London, and the \\ Royal Free Hospital, London
}

EDITORIAL COMMENT From these studies contraction of the longitudinal muscle of the oesophagus is considered to be the main factor causing elevation of the cardia into the chest with the development of a hiatus hernia.

THE RELATION BETWEEN REFLUX AND HERNIATION

According to generally accepted theory hiatal hernation is promoted by excess of intra-abdominal over intrathoracic pressure in the presence of an abnormally loose oesophageal hiatus. However, Pecora (1956) measured the hiatus with graduated feelers in 20 patients with hiatus hernia, and in only one did he find the size to exceed that found in 12 out of 20 apparently normal subjects.

In the course of operating on 187 patients with hiatus hernia I was struck with the fact that hernias of the short-oesophagus type were seldom more than an inch or two in length and the hiatus often seemed within normal limits. On the other hand all the paraoesophageal hernias were much larger than this and the hiatus was always conspicuously gaping. Indeed in two, almost the entire stomach was in the chest along with much great omentum, yet still the cardia remained near the diaphragm. It seemed evident that the two extreme types had been different from the start, and though largeness of the hiatus might contribute to herniation of the stomach, elevation of the cardia, when it occurred, required a separate explanation.

It has often been observed that whereas the common, sliding hiatus hernia is usually associated with oesophagitis, this is unusual in paraoesophageal hernia without oesophageal shortening. It was suggested that this might be because the shortening was due to scarring and was the result rather than the cause of the oesophagitis (Allison, Johnstone, and Royce, 1943). However, since fibrous shortening of the oesophagus is common only in long-standing cases, I advanced the hypothesis that it was spasm of the longitudinal muscle of the gullet that drew the cardia into the chest (Johnson, 1955). Muscle spasm is usual in inflammation, and since the longitudinal muscle of the gullet is attached indirectly to the base of the skull, its contraction must necessarily elevate the cardia.

The observation that oesophagitis could often be seen at oesophagoscopy and proved by biopsy in patients who showed no radiological evidence of herniation (Johnson, 1955, Conway-Hughes, 1956, Cross, Smith, and Kay, 1959) was consistent with the view that oesophagitis might precede and cause hiatal herniation of the short-oesophagus type. Moreover, oesophagitis without herniation has been more frequent among my younger patients with heartburn, a greater proportion of my older patients with this symptom having had established, sliding hernias. This gave further support to the proposition.

In postulating how reflux may occur without established herniation, reflux is considered under two headings: breakdown in the antireflux mechanism, and active opening of the cardia. Botha (1962) argued that the antireflux mechanism is almost certainly composite, depending for optimal efficiency upon mutual support by several elements. The elements are considered in turn.

\section{FAILURE OF THE ANTIREFLUX MECHANISM}

THE MUCOSAL PLUG When operating for haematemesis I sometimes search the stomach through a protoscope for a source of bleeding. On a number of occasions I have taken the opportunity to inspect the cardia. Before the days of curare it was usually totally occluded by folds of mucosa, presenting a smooth face, just as it does radiologically. No suggestion of a flap was ever seen. Allison (1956) believed the mucosal rosette to be held together by an oesophageal sphincter. Creamer (1955) suggested that muscularis mucosae acted pursestring-fashion, though this did not accord with the longitudinal direction of its fibres (Fig. 5). Botha (1962) considered that the folds of mucosa showed intrinsic 


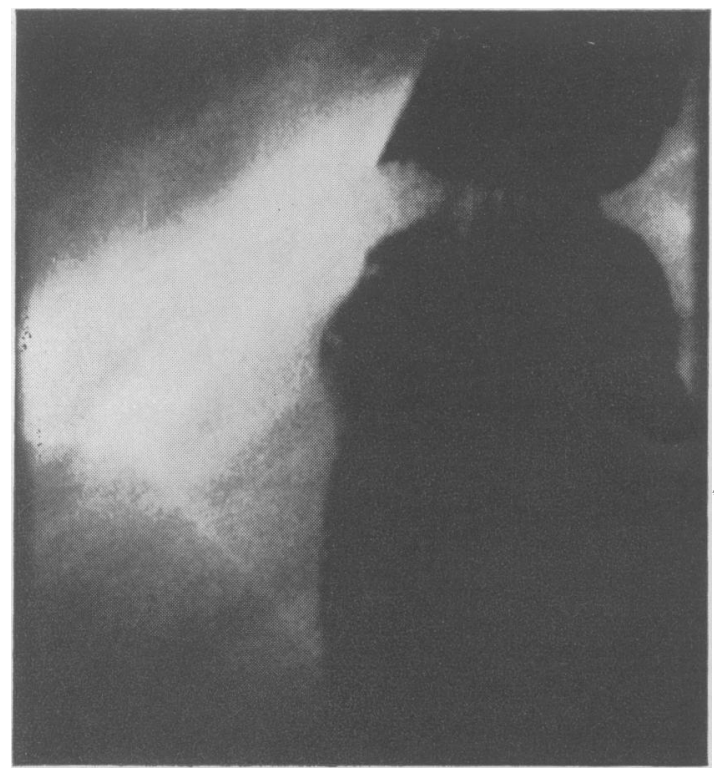

FIG. 1. Empty zone probably caused by a horizontal mucosal fold at the cardia (dog 1$)$.

motility. Radiological projection sometimes shows a sulcus outside a filling defect or empty zone (see Figure 1). The appearances suggest that local longitudinal contraction of muscularis mucosae has raised a circular ridge on the mucosa which, as it was crowded together, has broken up into a rosette (Figure.2).

Fluid pressure acts at right angles to the inner surface of a container. Therefore, however the mucosa is brought together, once it is so, intragastric pressure can no longer have any tendency to open
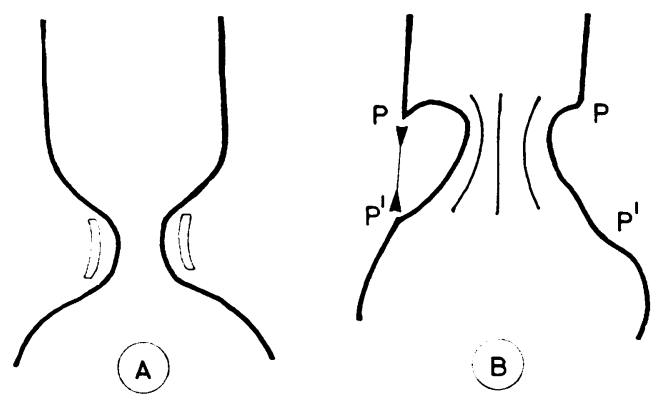

FIG. 2. A Appearance expected if empty zone had been produced by contraction of a ring of muscle.

$B$ Appearances actually seem, more suggestive of telescoping of mucosa by contraction of muscularis mucosae (the fibres of which run longitudinally) with raising of circular ridge on inside of gullet, this then breaking up into a rosette. the cardia. It can only press the mucosal plug as a whole against the closed oesophagus. Tenting or funnelling of the cardia, on the other hand, would reduce the effectiveness of mucosal apposition, letting in the wedge, as it were, of intragastric pressure. Such tenting is sometimes seen in radiographs of patients with oesophagitis but no detectable herniation (Figure 3).

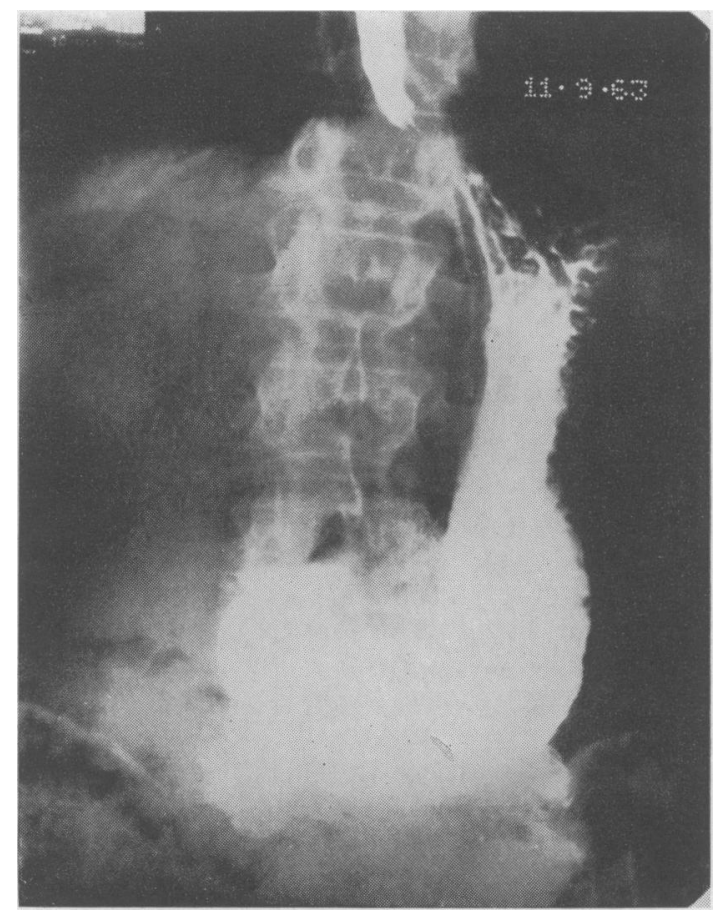

FIG. 3. A radiograph showing funnelling of the cardia and oesophageal reflux. This patient had severe reflux oesophagitis but no evidence of a hiatus hernia was reported. (There was also a duodenal ulcer.)

INFERIOR OESOPHAGEAL SPHINCTER Clear evidence of a functional sphincter is sometimes seen at the oesophagogastric junction, even though this is herniated into the chest (see Figure 1). Many workers have been puzzled by the fact that so little thickening of circular muscle is evident at the site.

Laplace's law implies that, for a given fluid pressure within it, the smaller the tube the less the tension in its wall. Anyone who has blown up a toy balloon should know this, for even when the balloon is tightly distended the inflator tube is still soft and easily, compressed. (This law is, of course, independent of the material in which the tension happens to be.) Though the stomach muscle needs to be sturdy, much thinner muscle suffices to over- 
come the same internal pressure in the relatively narrower abdominal oesophagus.

This element of the antireflux mechanism would be expected to become less efficient with advancing years and general loss of muscle tone. It is occasionally competent alone-but only occasionally.

Oesophago-gastric ANGLE Hiebert and Belsey (1961) suggested that funnelling of the cardia, short of herniation, might undermine the antireflux mechanism by obliterating the angle of His. Opinion is divided as to the importance of the oesophagogastric angle in resistance to reflux, but those who believe in it will observe that elevation of the cardia widens this angle.

ROLE OF THE ABDOMINAL OESOPHAGUS It is impossible to suck through a flaccid tube, for when one tries to do so the tube is collapsed by atmospheric pressure. The narrower the tube and the more vigorous the suction the more promptly does this occur. Similarly when the intra-abdominal oesophagus is 'sucked on' by subatmospheric pressure in the rigid, inspiratory thorax, it tends to be collapsed by intra-abdominal pressure (Johnson, 1954).

Some surgeons have held the abdominal oesophagus to be an invention of anatomists (Wells, 1954; Tanner, 1954; Allison, 1956). However, it has been shown radiologically and with the aid of metal markers, to be 1.4 to $3.3 \mathrm{~cm}$. long, in unanaesthetized normal subjects (Creamer, Harrison, and Pierce, 1959). These authors also showed experimentally that the 'empty segment' or 'sphincteric area' corresponded with the abdominal oesophagus. They attributed its closure to intra-abdominat pressure.

After discussing the point in his review of research on the antireflux mechanism, Atkinson (1962) dismissed it with the remark 'it is generally agreed that this flaccid segment could only act as an antireflux mechanism if intragastric does not exceed intra-abdominal pressure'. It is true that intraabdominal pressure could not alone close the cardia against intragastric pressure. There must certainly be and is a tiny contribution from circular muscle. However, intra-abdominal pressure is undeniably the source of $99 \%$ of intraluminal pressure in the abdominal oesophagus.

Intragastric pressure also has two components, that derived from intra-abdominal pressure which compresses the stomach as a whole, and that attributable to gastric muscle tone. The part produced by intra-abdominal pressure can undergo substantial elevation (Botha, 1962). However, it is balanced by compression of the abdominal oesophagus. Only the part produced by gastric tone has to be resisted by the rest of the antireflux mechanism.
This part is relatively small and less varying (Orr and Johnson, 1947). To oppose it, as explained above, should be an easy task for the mucosal plug supported by the circular muscle of the terminal oesophagus.

As pointed out by Cannon (1911) the peritoneal cavity behaves very much as though it were filled with water. In the erect position intra-abdominal pressure in the pelvis exceeds that in the subphrenic region by an amount about equivalent to a column of water extending from one region to the other. When a subject is inverted these pressure relationships are reversed. Intragastric pressure also correspondingly rises (Fyke, Code, and Schlazel, 1956). However, no tendency to reflux is observed in a normal person. The simultaneous and equal increase in compression of the abdominal oesophagus by intra-abdominal pressure which inversion also causes explains why no further strain is thrown on the sphincter. A similar matched rise of pressure both inside the stomach and outside the abdominal oesophagus is bound to occur on straining and in recumbency.

Most of the patients I have seen with hiatus hernia were either obese or had one of a variety of space-occupying lesions. Rigler and Eneboe (1935) reported that they had found hiatus hernias in the third trimester of pregnancy in $5 \%$ of primigravidae and $18 \%$ of pregnant multiparae. Factors such as abdominal compression, repeated straining, or increase of abdominal contents tend to extrude the abdominal oesophagus into the hiatus. It is my contention that it is at this point, short of demonstrable herniation of the stomach, that control of reflux is apt to break down (Figure 3). Oesophagitis and spastic oesophageal shortening then follow and help to promote a hernia.

\section{ACTIVE OPENING OF THE CARDIA}

It is commonly assumed that inhibition of the inferior oesophageal sphincter occurs during vomiting, and such inhibition has been demonstrated by Fyke et al. (1956) during swallowing. Dornhorst, Harrison, and Pierce (1954) observed that eructation might follow a rise of intragastric pressure so small that it could not be judged to have forced the valve. They considered that active opening of the cardia must have taken place.

NATURE OF THE OPENING MECHANISM At the beginning of the century, Schreiber (1904) gave a vivid account of how the mucosa which plugs the cardia in the rabbit is drawn up and parted to allow fluid to pass through. Nauta (1956) observed that the cardia is lifted during swallowing in the dog. 
Torrance (1958) reported that contraction of the longitudinal muscle of the oesophagus is accompanied by opening of the cardia in the cat, and this may be effected by vagal stimulation or by induced vomiting. Ingelfinger (1958) remarked that the cardia is elevated during vomiting in man.

\section{EXPERIMENTAL EVIDENCE}

OBSERVATIONS ON THE RABBIT The appearances in the rabbit reported by Schreiber (1904) were confirmed. In the resting (anaesthetized) condition the cardia was not easily seen. Stimulation of the vagus nerve in the neck caused the mucosa to be drawn up into a tent, and a gaping lumen appeared right through into the supradiaphragmatic oesophagus. When the eosophagus was isolated in the neck, encircled by a tape, and pulled upon, exactly the same changes at the cardia were shown. Even after removal of the stomach and oesophagus similar opening of the cardia could be effected by pulling on the oesophagus. It appeared that the longitudinal muscle of the gullet was responsible for opening of the cardia (Figure 4).

HISTOLOGICAL STUdies Since the size of a muscle serves as an index of functional requirements, it was judged that the degree of development of this layer in the gullets of animals of different species might show some correlation with vomiting habits. Accordingly the oesophagus of some selected species was compared from this point of view.

In Fig. 5 are shown sections of the gullets of a cat and a rat, chosen because they lie at functional extremes. Whereas cats are proverbial vomiters rats do not vomit at all. It is seen at once that the proportion of longitudinal muscle is quite different and considerably larger in the animal with frequent need of an opening mechanism. The position of striped, voluntary elements in the gullets of ruminants suggested a capacity to open the 'cardia' voluntarily.

HIATUS HERNIA AND Vomiting Dey, Gilbert, Trump, and Roskelley (1946) found that stimulation of various abdominal organs in anaesthetized dogs, particularly the biliary apparatus, caused oesophageal spasm and shortening. A hiatus hernia appeared. On the hypothesis under consideration the oesophageal spasm may have represented vigorous opening of the cardia and been part of the vomiting reflex.

The following investigation was undertaken to ascertain whether any relationship between vomiting and hiatus hernia could be established experimentally.

Induced vomiting in the $\operatorname{dog} \mathrm{A}$ dog was given $200 \mathrm{ml}$. of barium emulsion by tube and funnel. The animal then lay on the $x$-ray table for screening and cine-radiography, and received an intravenous injection of $3.25 \mathrm{mg}$. of apomorphine. One and a half minutes later breathing became rapid and deep and the spine was arched. The dog then retched repeatedly. Each time a barium shadow was seen to rise a short way into the chest but was checked sharply, always at the same place (Figure 6). On the fourth occasion the barium passed right up the gullet and the dog vomited. (No restraint was required, possibly because of the tranquillizing effect of the apomorphine.)

The level at which the barium stopped during ineffective retching resembled a herniated cardia. However, in order to establish this beyond question a second dog was specially prepared before filming.

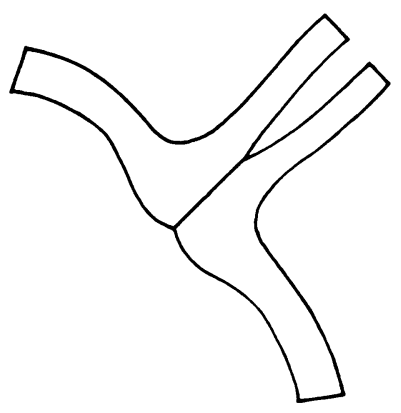

(A)
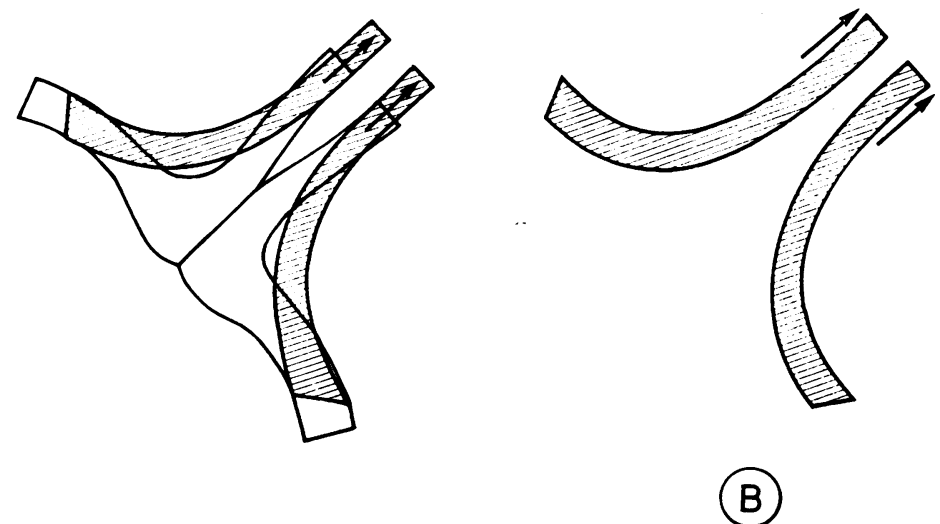

FIG. 4. The effect of elevation of the cardia on the mucosal plug. It also obliterates the abdominal oesophagus and alters the angle of His. 
FIG. 5a

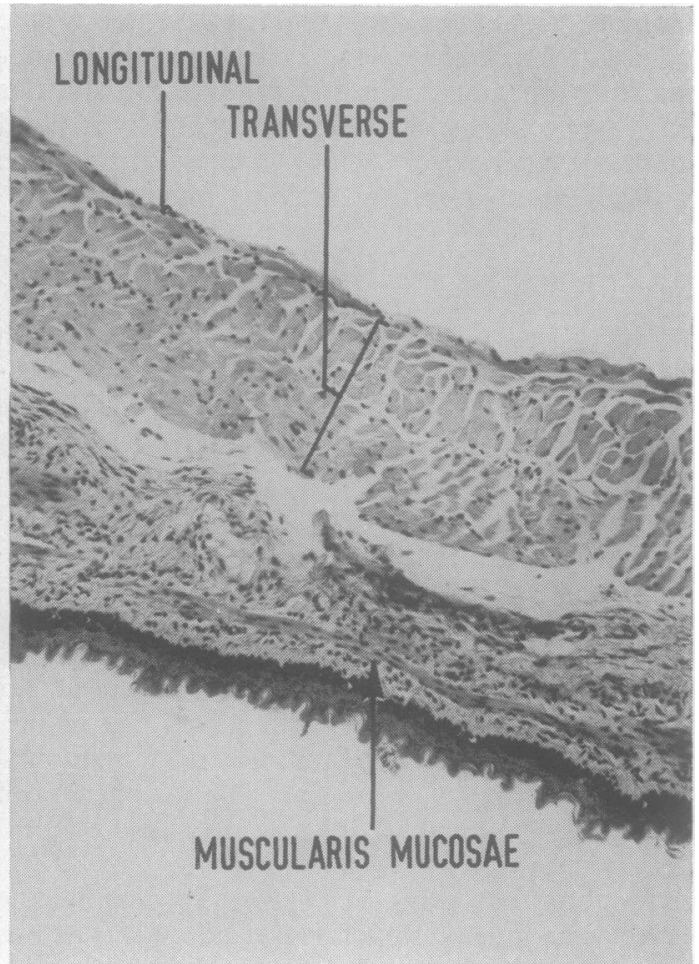

FIG. $5 b$

FIG. 5. Transverse section of the gullet of a cat (a), and longitudinal section of the gullet of a rat (b), to show the relative amounts of longitudinal muscle. The cat vomits freely, the rat not at all. Also note the longitudinal direction of the fibres of muscularis mucosae, which is the same as in man.

At open thoractomy differently shaped silver clips were fixed near the margin of the oesophageal hiatus of the diaphragm, and on the lowest inch of

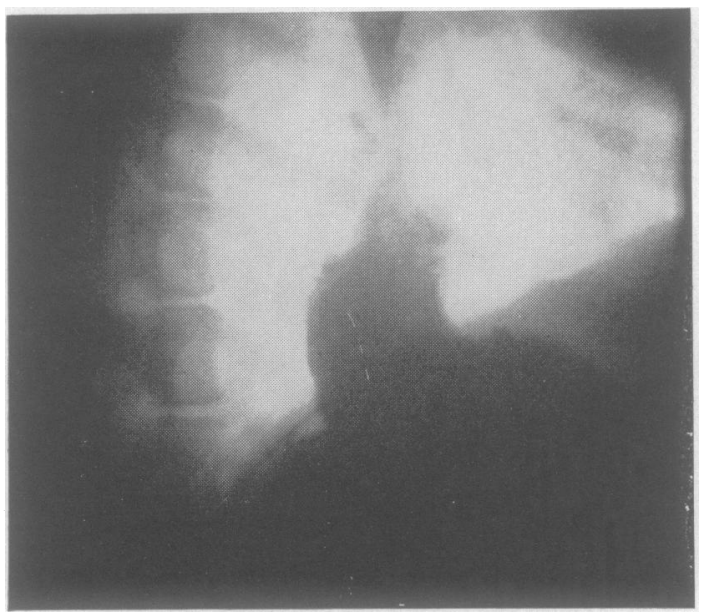

FIG. 6. Appearances on retching in the first dog. the gullet. The four straight pieces of silver wire were fixed with nylon sutures to the pleura covering the lowest part of the oesophagus, the lowest pair being at the level at which the visceral pleura was reflected onto the upper surface of the oblique hiatal margin of the diaphragm. Folded clips were sewn to the pleura covering the upper surface of the muscle of the hiatus. Figure 7 represents the diaphragm of this dog before vomiting, showing the position of the clips. In Figure 8, taken just before injection of apomorphine, the hiatal markers are just level with the lowest pair on the oesophagus.

Damage to muscle was deliberately avoided in fixing these markers so as to interfere as little as possible with function. Though it was hoped that the clips would to some extent reflect movement of the oesophagus in relation to the hiatus, it was not expected that they would follow such movement exactly. Rather was it judged that most of the movement, if any, would be of the oesophagus within the pleura.

After recovery the dog underwent the same 


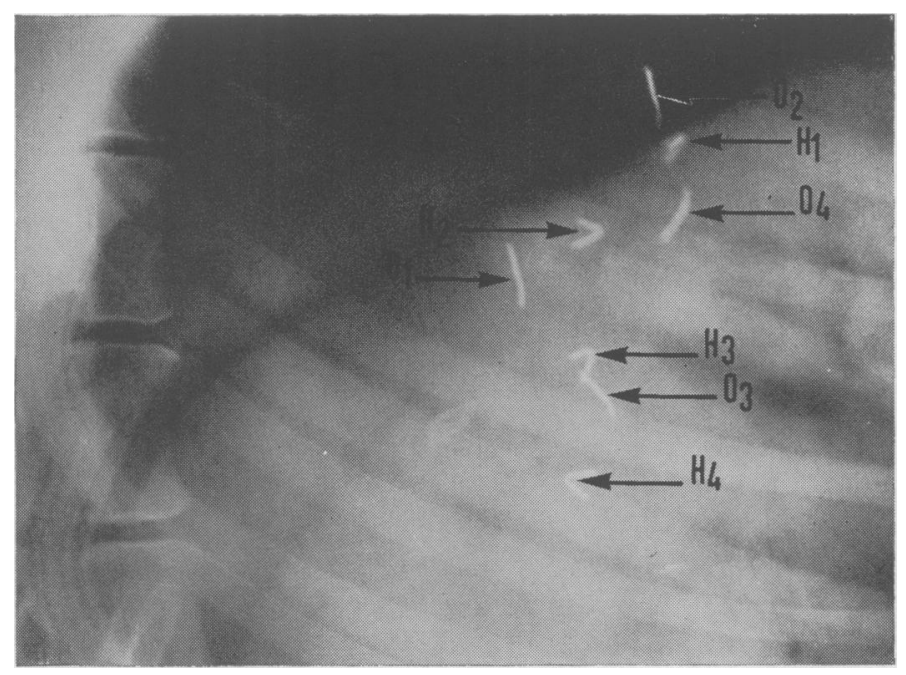

FIG. 7. Ordinary still radiograph of the cardia of the second dog, to show the position of the markers. Quiet breathing; expiration. $\bigcirc, 1-4 ;$ Straight markers sewn to pleura on oesophagus. $H, 1-4$; Folded markers sewn to pleura covering hiatal muscle. The pleura dips into the hiatus during expiration and the lowest oesophageal markers are below those on top of the hiatal muscle.
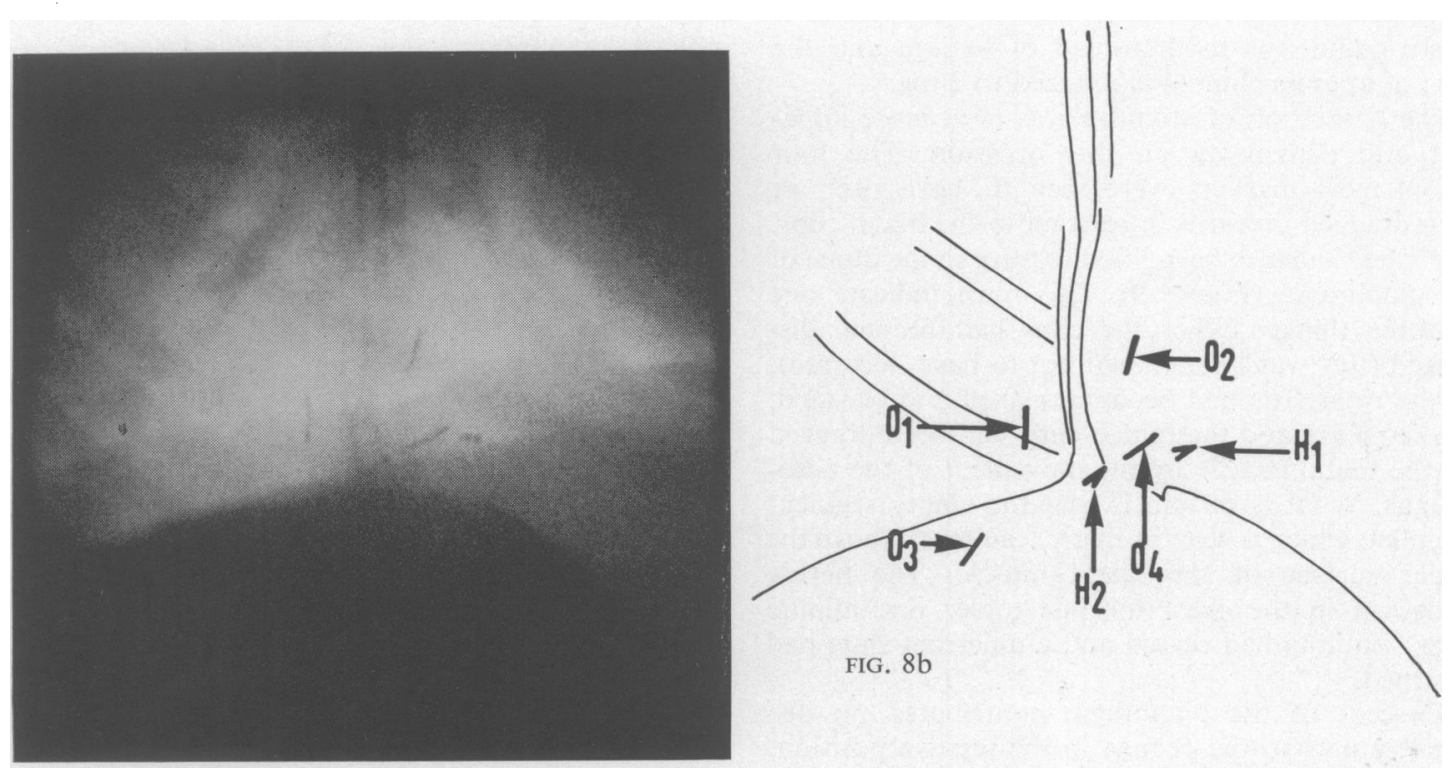

FIG. $8 \mathrm{a}$

FIG. 8a. One frame of a cine-radiograph of the second dog during inspiration and before vomiting to show the position of the markers.

FIG. 8b. Tracing of Fig. 8a with annotation of markers as in Figure $7 . \mathrm{H}_{3}$ and $\mathrm{H}_{4}$ are not seen. 


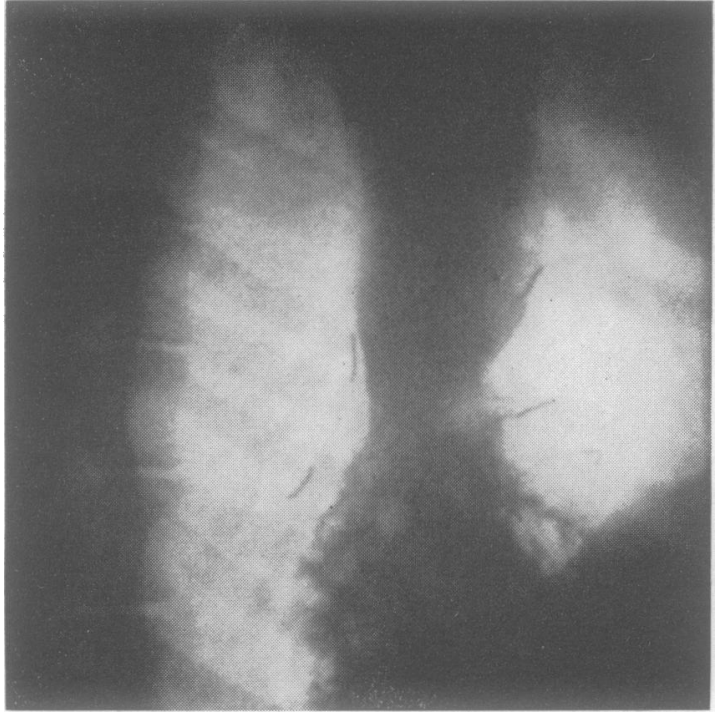

FIG. 9a
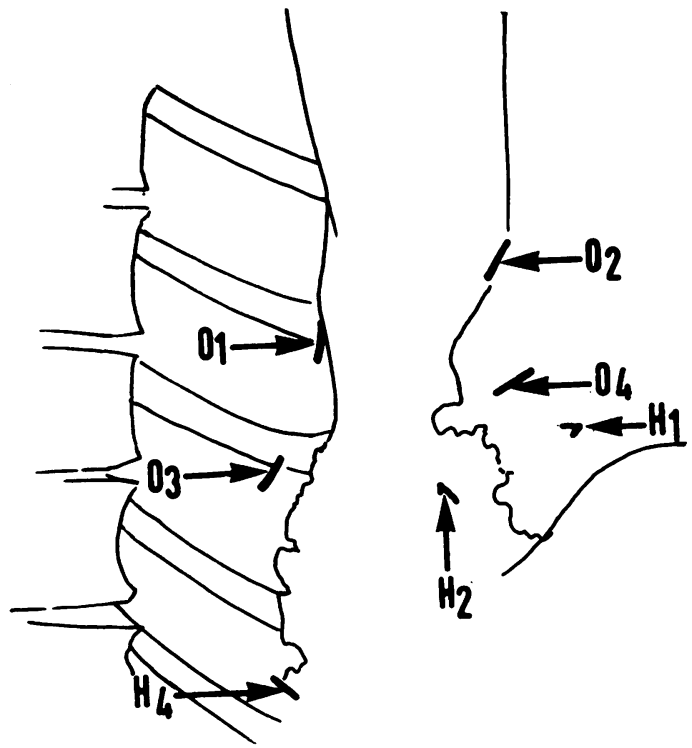

FIG. $9 \mathrm{~b}$

FIG. 9a. The second dog. During vomiting both sets of markers have risen relative to the dome of the diaphragm. The $O$ markers have also risen relative to the $H$ markers.

FIG. 9b. Tracing of Fig. $4 a$ with annotations as in Figure 7.

procedure as the last one, except that this time Gastrografin was used instead of barium and the dose of apomorphine was reduced to $2 \mathrm{mg}$.

The herniation of stomach was even more apparent and convincing on this occasion. The four oesophageal markers were seen to have risen or been dragged upwards in relation to the hiatal clips. The latter had also been lifted relative to the dome of the diaphragm (Figure 9). This must indicate one of three things: either the clips had become dislodged (this was later shown not to have occurred), or the right crus had become relaxed and elevated, or the pleura and the folded clips had been dragged off the hiatal muscle by the movement of the oesophagus. What is certain is that the empty segment of gullet, which is seen in many frames, is above the upper surface of the hiatal muscle. The hernia remained in the chest for just under one minute after vomiting had ceased and a quiescent state had returned.

Descent of the diaphragm contributed no discernible part to the change in the relative positions of the hiatus and the cardia, all of which appeared to have resulted from oesophageal shortening. Indeed, wide respiratory excursions occurred while a piece of stomach was herniated into the chest, and the length of the oesophagus always adjusted itself to the position of the diaphragm. The hernia under- went no obvious change in size as it rose and fell with the diaphragm.

The experiment was repeated with a third dog with almost identical results, the only difference being that this time the opaque medium did not check, as previously, at the herniated cardia, but passed right up the gullet at the first effort. This time the markers consisted of $5 \mathrm{~mm}$. lengths of fine polythene tube filled with barium sulphate powder and sealed. These were buried in the muscle of the hiatus and in the muscular wall of the oesophagus. One cine-radiograph of this third dog was made before operation and another after marking (Figs. 10 and 11), so that any change resulting from the operation could be noted. None was in fact seen.

The experiment was repeated in a fourth dog, anaesthetized and with both abdomen and chest open, to ascertain whether elimination of pressure difference affected the result. The hernia appeared as previously (Fig. 12), and must therefore have resulted from oesophageal traction.

DEMONSTRATION OF THE ROLE OF THE ABDOMINAL OESOPHAGUS Many people find the effect of ambient pressure on a soft tube hard to grasp or even to credit. The following experiment is therefore reported by way of illustration:

A 2 in. segment of jejunum was used to construct 


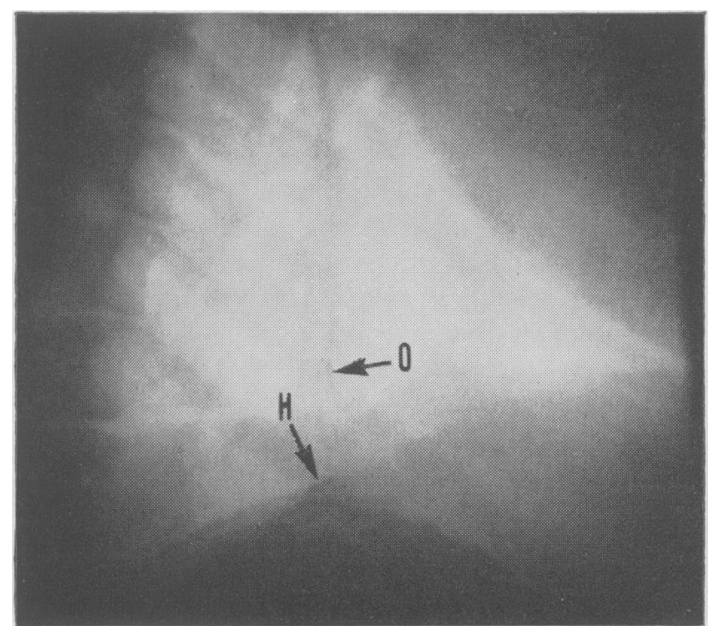

FIG. 10

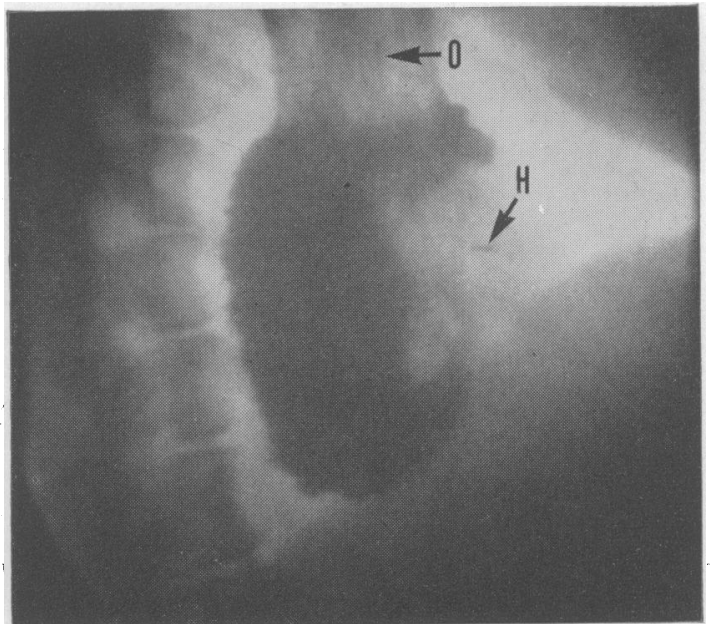

FIG. 11

FIG. 10. The third dog before vomiting. $O$ marker buried in longitudinal muscle of oesophagus. $H$ marker buried in muscle of hiatus.

FIG. 11. The third dog during vomiting. Markers as in Figure 10.

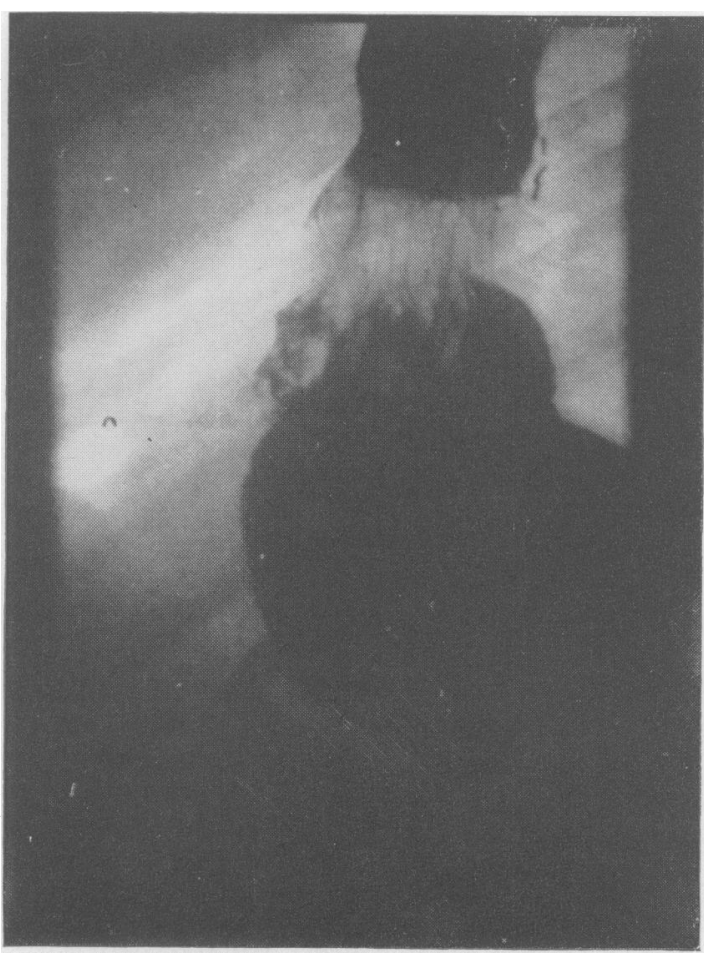

FIG. 12. The fourth dog anaesthetized and with both abdomen and chest open, after injection of apomorphine. a gastrostomy on a dog. About $200 \mathrm{ml}$. of milk was introduced into the stomach and $1.7 \mathrm{mg}$. of apomorphine was given intravenously. When the dog vomited the feed none leaked from the gastrostomy.

On the following day a medium-sized, gum-elastic catheter was introduced into the stomach via the gastrostomy. It was a loose fit, allowing a second catheter to be passed easily alongside. The meal and apomorphine were repeated. This time, as the dog vomited, milk also squirted out of the catheter under considerable pressure but none at all escaped alongside the catheter. As the diaphragm descended intragastric pressure rose to over $800 \mathrm{~mm}$. $\mathrm{Hg}$. But this was due to an equal rise in intra-abdominal pressure. Compression of the bowel segment was increased similarly. Any small excess of intragastric over intra-abdominal pressure was controlled by tone in the circular muscle of the segment of small bowel, just as it is in the abdominal oesophagus when reflux is being opposed.

RELATED CLINICAL OBSERVATIONS Formerly physicians used to describe 'flatulent dyspepsia' as pathognomonic of gallstones. More recently it has been suggested that the belching and heartburn are really due to an associated hiatus hernia. Gallstones were found in $12 \%$ of the 156 of my patients who were operated on abdominally for hiatus hernia. Moreover, two of them had chronic cholecystitis 
and many small stones, but negative radiographs. The biliary lesions would have remained undiagnosed had these patients undergone thoracic operations for hernia. Two others operated on abdominally for hiatus hernia had no gallstones then, but gallstones were present when the patients were reoperated on for recurrent hernia four and five years later.

The high incidence of gallstones among hiatus hernia patients suggests a causative connection. The observations of Dey et al. (1946), quoted above, show how biliary stimulation may promote herniation in the dog, and my own work favours the view that this involves the vomiting reflex. However, the incidence of hiatus hernia among patients with gall-bladder disease and 'flatulent dyspepsia' has been only $5 \%$ in my series, though some of the other gall-stone patients who had heartburn were oesophagoscoped and found to have evidence of oesophagitis. An alternative explanation for their symptoms was that they were due, not to undiagnosed hiatus hernia, but to repeated active opening of the cardia, and that this represented part of the vomiting reflex.

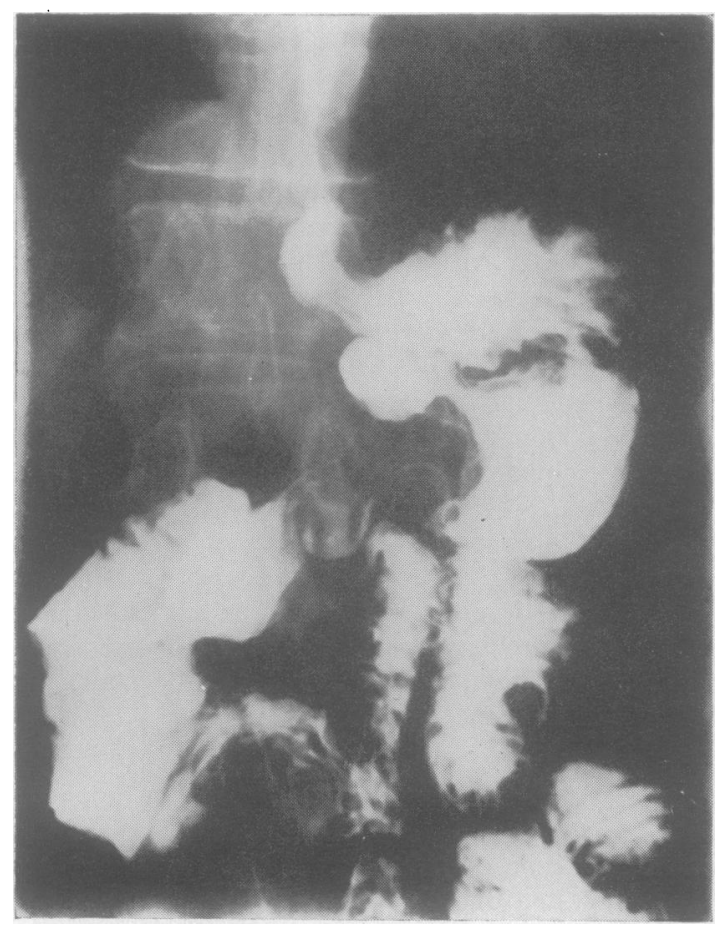

FIG. 13. A radiograph showing the triad, pre-pyloric or duodenal ulcer, plus large lesser curve ulcer, plus hiatus hernia (confirmed at operation). Unlike those of some triads these elements are held to be causally interrelated.
Peptic ulcers have been found 31 times in my series of 187 hiatus hernia operations $(17 \%)$. Though 10 hernias were discovered at laparotomy in the course of operations for ulcers, 21 others were seen in radiographs before operation. (Two patients also had gallstones.)

A juxtapyloric ulcer with a dilated stomach was the more usual combination, and though seven patients had high gastric ulcers, five of these had evidence of old duodenal lesions as well. A sixth had adult pyloric hypertrophy. Botha (1962) observed that reflux is more apt to occur when the stomach is distended, and it may well be that when hiatus hernia is found in association with duodenal ulcer it is the hernia that is secondary to the ulcer, to gastric retention, and to the reflux oesophagitis which I have commonly found by oesophagoscopy in patients with this disease.

It has been previously reported (Johnson, 1955, 1957) that duodenal or pyloric ulcers, particularly with gastric retention, are apt to promote a special kind of secondary gastric ulcer in addition. Patients who develop these ulcers differ in important respects from those who harbour other gastric ulcers (Johnson, Love, Rogers, and Wyatt, 1964). Nearly all the gastric ulcers found in association with hiatus hernias were judged to be of this secondary type. Indeed the triad of duodenal or juxtapyloric ulcer with lesser curve ulcer and also sliding hiatus hernia would seem to be sufficiently frequent to justify recognition as a clinical entity. A radiograph of one is shown in Figure 13. There have been five instances of this triad among my own patients and I have seen three more among those of others.

\section{CONCLUSIONS}

The 'physiological empty segment' closes when the sum of forces acting inwards at right angles to its lining exceeds those acting in the opposite direction, and vice versa. When the cardia is open the latter force equals intragastric pressure but, by Laplace's law, the tension in the circular muscle of the terminal oesophagus is also proportional to its internal diameter. When the cardia is closed and plugged by mucosa this equals zero and the tension in circular muscle required to keep the 'empty segment' empty is therefore infinitesimally small.

The closing forces include that due to oesophageal circular muscle and also intra-abdominal pressure which, is close to atmospheric pressure at this level in the erect position, and rises in step with intragastric pressure on straining or in recumbency. Since the circular muscle is never called upon to exert more than trivial force it is naturally relatively frail.

Active opening of the cardia is a function of the 
longitudinal muscle of the gullet, contraction of which tents the cardia and the mucosal plug, also obliterating the abdominal oesophagus. The whole of intragastric pressure then meets the sphincter supported only by intrathoracic instead of intraabdominal pressure. Moreover, during swallowing, eructation, and vomiting sphincteric tone is inhibited.

Though largeness of the hiatus may cause herniation of the stomach, elevation of the cardia when this occurs as well is caused by spasm of the longitudinal muscle. This can be a result of preceding oesophagitis due to extrusion of the abdominal oesophagus and reflux, or it may represent active opening of the cardia and form part of the vomiting reflex. Active opening occurs in gall-bladder disease and may be the reason for the characteristic eructation and heartburn as well as for the hiatus hernia which commonly complicates this condition.

\section{SUMMARY}

The hypothesis is advanced that the factor which determines whether or not the cardia is elevated into the chest in hiatus hernia is contraction of the longitudinal muscle of the oesophagus. It is suggested that such spasm is apt to be promoted by oesophagitis, and that reflux may precede frank herniation of the short oesophagus type. Technical reasons are given for believing that obliteration of the abdominal oesophagus is enough to undermine a part of the antireflux mechanism which is particularly important during straining and in recumbency.

Evidence of active opening of the cardia is quoted and it is pointed out that this must form a part of the vomiting reflex. The mechanism of opening of the cardia is considered experimentally and by direct observation. It is also shown to involve elevation of the cardia, and even a large hiatus hernia during vomiting in dogs.

Excessive stimulation of the vomiting reflex is advanced as a factor in the production of sliding hiatus hernia. Clinical evidence is reported which accords with this suggestion.

It is a pleasure to acknowledge the generous help I have received from many colleagues, particularly Professor R. E. Steiner and Dr. J. W. Laws and the staff of the Department of Radiology; also Professor C. V. Harrison, of the Department of Morbid Anatomy, and Professor R. B. Welbourn, of the Department of Surgery, all at the Postgraduate Medical School; also Dr. G. B. D. Scott, of the Department of Morbid Anatomy at the
Royal Free Hospital, and Mr. R. N. Fiennes, pathologist to the Zoological Society. My grateful thanks are also due to Mr. Robson, senior technician, and the staff of the Experimental Surgical Unit at the Postgraduate Medical School, and to Mr. Phillips, senior photographer at the Royal Free Hospital. The work was supported by a generous grant from the Royal Free Hospital Endowment Funds.

\section{REFERENCES}

Allison, P. R., Johnstone, A. S., and Royce, G. B. (1943). Short oesophagus and simple peptic ulceration. J. thorac. Surg., 12 , 432-457.

Allison, P. R. (1956). Function and dysfunction at the cardia. Bull. Johns Hopk. Hosp., 99, 182-189.

Atkinson, M. (1962). Mechanism protecting against gastro-oesophageal reflux: a review. Gut, 3, 1-15.

Botha, G. S. M. (1962). The Gastro-oesophageal Junction. Churchill, London.

Cannon, W. B. (1911). The Mechanical Factors of Digestion. Arnold, London.

Conway-Hughes, J. H. L. (1956). Oesophageal reflux-an analysis of 453 consecutive barium meal examinations. Brit. J. Radiol., 29, 331-334.

Creamer, B. (1955). Oesophageal reflux and the action of carminatives. Lancet, 1, 590-592.

Harrison, G. K., and Pierce, J. W. (1959). Further observations on the gastro-oesophageal junction. Thorax, 14, 132-137.

Cross, F. S., Smith, G. V., Jr., and Kay, E. B. (1959). The surgical treatment of peptic esophagitis. J. thorac. cardiovasc. Surg., 38, 798-813.

Dey, F. L., Gilbert, N. C., Trump, R., and Roskelley, R. C. (1946). Reflex shortening of the esophagus in the experimental animal with the production of esophageal hiatus hernia. J. Lab. clin. Med., 31, 499-506.

Dornhorst, A. C., Harrison, K., and Pierce, J. W. (1954). Observations on the normal oesophagus and cardia. Lancet, 1, 695-698.

Fyke, F. E., Jr., Code, C. F., and Schlegel, J. F. (1956). The gastroesophageal sphincter in healthy human beings. Gastroenterologia (Basel), 86, 135-150.

Hiebert, C. A., and Belsey, R. (1961). Incompetency of the gastric cardia without radiologic evidence of hiatal hernia. $J$. thorac. cardiovasc. Surg., 42, 342-350.

Ingelfinger, F. J. (1958). Esophageal motility. Physiol. Rev., 38, 533-584.

Johnson, H. D. (1954). Contribution to Discussion on hiatus hernia. Meeting of Ass. Surg. G.B. \& I., Leeds.

- (1955). The special significance of concomitant gastric and duodenal ulcers. Lancet, 266-270.

- (1957). The classification and principles of treatment of gastric ulcers. Ibid., 2, 518-520.

Love, A. H. G., Rogers, N. C., and Wyatt, A. P. (1964). Gastric ulcers, blood-groups, and acid secretion. Gut, 5, 402-411.

Nauta, J. (1956). The closing mechanism between the oesophagus and the stomach. Gastroenterologia (Basel), 86, 219-232.

Orr, I. M., and Johnson, H. D., (1947). Vagal resection in the treatment of duodenal ulcer. Lancet, 2, 84-89.

Pecora, D. V. (1956). Observations on the pathologic physiology of the lower esophagus in sliding hiatus hernia with comments on surgical treatment. Ann. Surg., 143, 459-464.

Rigler, L. G., and Eneboe, J. B. (1935). The incidence of hiatus hernia in pregnant women and its significance. J. thorac. Surg., 4, 262-268.

Schreiber, J. (1904). Ueber den Schluckmechanismus. Hirschwald, Berlin.

Tanner, N. C. (1954). Contribution to Discussion on hiatus hernia. Meeting of Ass. Surg. G.B. \& I., Leeds.

Torrance, H. B. (1958). Studies on the mechanism of gastro-oesophageal regurgitation. J. roy. Coll. Surg. Edinb., 4, 54-62.

Wells, C. A. (1954). Contribution to Discussion on hiatus hernia. Meeting of Ass. Surg. G.B. \& I., Leeds. 